\title{
Influence of Load on Reliability of Storage Area Networks
}

\author{
Guixiang Lv \\ Department of Electrical and Computer Engineering, \\ University of Massachusetts, Dartmouth, MA, USA. \\ E-mail: glv@umassd.edu \\ Liudong Xing \\ Department of Electrical and Computer Engineering, \\ University of Massachusetts, Dartmouth, MA, USA. \\ Corresponding author: 1xing@ umassd.edu
}

(Received on August 11, 2021; Accepted on October 12, 2021)

\begin{abstract}
During the coronavirus pandemic, telecommuting is widely required, making remote data access grow significantly. This requires highly reliable data storage solutions. Storage area networks (SANs) are one of such solutions. To guarantee that SANs can deliver the desired quality of service, cascading failures must be prevented, which occur when a single initial incident triggers a cascade of unexpected failures of other devices. One such incident is the data loading/overloading, causing the malfunction of one device and further cascading failures. Thus, it is crucial to address influence of data loading on the SAN reliability modeling and analysis. In this work, we make contributions by modeling the effects of data loading on the reliability of an individual switch device in SANs though the proportional-hazards model and accelerated failure-time model. Effects of loading on the reliability of the entire SAN are further investigated through dynamic fault trees and binary decision diagrams-based analysis of a mesh SAN system.
\end{abstract}

Keywords- Storage area network, Cascading failure, Load, Proportional-hazards model, Accelerated failure-time model.

$\begin{array}{ll}\begin{array}{ll}\text { Acronyms } \\ \text { AFTM }\end{array} & \text { Accelerated failure-time model } \\ \text { BDD } & \text { Binary decision diagram } \\ \text { DFT } & \text { Dynamic fault tree } \\ \text { FT } & \text { Fault tree } \\ \text { PDF } & \text { Probability density function } \\ \text { PHM } & \text { Proportional-hazards model } \\ \text { SAN } & \text { Storage area network } \\ & \\ \text { Notations } & \text { Baseline or initial failure rate } \\ \lambda_{0} & \text { Failure rate } \\ \lambda & \text { Load model parameter } \\ \alpha & \text { Baseline load or initial load } \\ L 0 & \text { Load on the system component } \\ L & \text { System reliability when the load on the component under study is } L \\ R_{\text {sys }}(L) & \text { Component reliability function at time } t \text { when the load on the component is } L \\ R(t ; L) & \text { Component failure function at time } t \text { when the load on the component is } L \\ F(t ; L) & \text { Component failure rate at time } t \text { when the load on the component is } L \\ h(t ; L) & \text { Component time-independent failure rate when the load on the component is } L \\ \lambda(L) & \text { Multiplicative factor used in the load model when the load is } L \\ \phi(L) & \end{array}$




\section{Introduction}

Telecommuting is widely required during the coronavirus pandemic, making remote data access grow significantly (Bright and Raschid, 2000; Hutanu et al., 2010). There are two major options for network-based storage solutions: storage area networks (SANs) and network-attached storage (Levens, 2021). An SAN is a high-speed network that interconnects storage devices, computer servers, and a management layer facilitating the access to any shared storage device by any server within the network, i.e., any-to-any interconnections of servers and storage devices (Honma et al., 2004; Katal et al., 2012; Xing et al., 2017). A network-attached storage is a single storage device connected to a network, providing file-based data storage and retrieval services to users and clients over Ethernet (Gibson and Rodney, 2000).

Due to benefits and features of SANs (e.g., delivering high throughput and low latency, relieving stresses on a local area network, and being the unique solution for demanding applications requiring concurrent shared access), SANs have two-thirds of the total networked storage market and are popular to be used by many famous enterprises, such as IBM, Tintri, NetApp, etc. (Levens, 2021). For the robust operation of SANs (or stably delivering the desired quality of service), reliability modeling and analysis are conducted as a crucial step in the SAN design and maintenance.

A challenge to achieve high reliability of SANs is to prevent or mitigate cascading failures. Cascading failures widely exist in diverse applications such as power grid systems, transportation network systems, infrastructure systems, smart home systems, and so on (Xiao and Yeh, 2011; Yang et al., 2016; Chen et al., 2019). Due to the physically or logically interdependent configuration of system devices, a single initial incident triggers a cascade of unexpected failures of other devices (Xing, 2021). Many causes can lead to the occurrence of cascading failures, for example, the malfunction of one device, overload, operator errors, physical attacks, cyber-attacks, and other environmental factors (Timashev, 2019; Amazon, 2020; Mishra et al., 2020). Among them, overload is the most common one. For example, in an SAN system containing switches for data transmission between servers and storage arrays, the overloading of one switch can fail this switch and lead to the redistribution of its workload to other switches, which may further cause overloads on those switches in a domino way. Thus, it is crucial to model effects of loading in the SAN reliability modeling and analysis, which can aid in the design of mitigation strategies against cascading failures caused by overloading.

Some research efforts have been expended in the reliability modeling and analysis of SAN systems. For example, in Xing et al. (2017), dynamic fault trees and network graph theory coupled with binary decision diagrams were proposed for evaluating the reliability of the mesh SAN with perfect and imperfect links, respectively. In Qiu et al. (2005), analytical models were developed for assessing and comparing the reliability and availability related metrics of optical-based solutions and IP-based solutions for SAN extensions. In Uwaechia and Akinsanmi (2013), the SAN model coupled with the redundancy array of independent disks (RAID) technology was designed and its reliability was evaluated for a data network repository. In Jiang et al. (2007), a fuzzy logic and Markov model-based method was developed for reliability analysis of an iSCSI protocol-based fault tolerant SAN. In Shetty (2002), the reliability block diagrams-based method was used to assess availability and reliability metrics of SANs configured with Dell servers, storage arrays and Fiber Channel switches. However, all these works fail to consider the effects of loading in the SAN reliability modeling and analysis. In this work, we make contributions by modeling the effects of data loading on the reliability of an individual switch device in SANs though two different loadfailure models: the proportional-hazards model and accelerated failure-time model. Effects of 
loading on the overall reliability of the SAN are further investigated through dynamic fault trees and binary decision diagrams-based analysis of a mesh SAN system.

The remainder of this paper is organized as follows: Section 2 presents models for describing the relationship between the load and failure behavior of an SAN device. Section 3 depicts an illustrative example of a mesh SAN system. Section 4 performs reliability modeling and analysis for the example SAN system. Section 5 examines effects of loading on the switch reliability and the entire SAN reliability. Section 6 gives conclusions and directions for future work.

\section{Load-Failure Rate Relationship Models}

To model the relationship between the loading and failure behavior of a device in the SAN, the proportional-hazards model, and the accelerated failure-time model (Levitin and Amari, 2009) are applied.

\subsection{Proportional-Hazards Model (PHM)}

PHM is a class of survival models in statistics. It is a regression model used in diverse applications such as medical research (Singh and Mukhopadhyay, 2011), the prediction of bank failures (Lane et al., 1986), and the reliability field (Dale, 1985; Mohammad et al., 2013).

PHM assumes that the hazard (failure) rate of a component is the product of both a baseline hazard rate and a multiplicative factor based on the values of a set of conditions. Note that the hazard rate can be a function of time $t$. Therefore, the component failure rate at time $t$ and under load $L$, denoted by $h(t ; L)$, can be obtained as $(1)$, where $h_{0}(t), z$, and $\alpha$ represent the baseline hazard rate function, a set of loads acting on the component, and vectors of the unknown parameters in the PHM, respectively.

$h(t ; L)=h_{0}(t) e^{z \alpha}$

When there is only one type of load, i.e., $z=L$, and $h_{0}(t)$ is a constant (independent of time), i.e., $h_{0}(t)=\lambda_{0}$, the failure rate $h(t ; L)$ is independent of time and can be obtained as

$h(t ; L)=\lambda(L)=\lambda_{0} e^{L \alpha}$.

\subsection{Accelerated Failure-Time Model (AFTM)}

AFTM describes a situation where the biological or mechanical life history of an event is accelerated. It has been applied in fields such as the counting processes in the bladder cancer study (Lin et al., 1998), the data description in the influenza and in acute liver failure (Kay and Kinnersley, 2002; Khanal et al., 2014).

In AFTM, a component's reliability, denoted by $R(t ; L)$, is expressed as (3).

$R(t ; L)=R_{0}(t \phi(L))$

where, $R_{0}$ represents the survival/reliability function of an arbitrary distribution, $t$ represents the mission time, and $\phi(L)$ denotes a multiplicative factor. The multiplicative factor $\phi(L)$ is also referred to as an acceleration/deceleration factor used to reflect different stress levels experienced under different loads. When there is only one type of load, $\phi(L)$ can be determined based on either the power law or the exponential law, which are expressed as (4) with $\alpha$ being the effect parameter. $\phi(L)= \begin{cases}L^{\alpha}, & \text { Power law } \\ e^{L \alpha}, & \text { Exponential law }\end{cases}$ 
The component failure rate, denoted by $\lambda(L)$, has a different format based on the expression of $\phi(L)$. Next, we derive the component failure rate under the power load and the exponential law.

\subsubsection{Failure Rate under the Power Law}

When the baseline time-to-failure distribution is exponential and the multiplicative factor at $L_{0}$ (i.e., $\left.\phi\left(L_{0}\right)\right)$ follows the power law, $R\left(t ; L_{0}\right)$ is obtained as Eq. (5) by applying Eq. (3). The failure function, denoted by $F\left(t ; L_{0}\right)$, is obtained as Eq. (6). Consequently, the probability density function, $f\left(t ; L_{0}\right)$, is obtained as the derivative of the failure function as shown in Eq. (7).

$R\left(t ; L_{0}\right)=R_{0}\left(t \phi\left(L_{0}\right)\right)=e^{-\lambda\left(t \phi\left(L_{0}\right)\right)}=e^{-\lambda t L_{0}{ }^{\alpha}}$

$F\left(t ; L_{0}\right)=1-R\left(t ; L_{0}\right)=1-e^{-\lambda t L_{0}{ }^{\alpha}}$

$f\left(t ; L_{0}\right)=\frac{d F\left(t ; L_{0}\right)}{d t}=L_{0}{ }^{\alpha} \lambda e^{-\lambda t L_{0}{ }^{\alpha}}$

Therefore, the failure rate at load $L_{0}$, which is independent of time and denoted by $\lambda\left(L_{0}\right)$, can be obtained as Eq. (8).

$\lambda\left(L_{0}\right)=\frac{f\left(t ; L_{0}\right)}{R\left(t ; L_{0}\right)}=L_{0}{ }^{\alpha} \lambda$

Similarly, we have the failure rate at any load $L$ as

$\lambda(L)=\frac{f(t ; L)}{R(t ; L)}=L^{\alpha} \lambda$.

The relationship between $\lambda(L)$ and $\lambda\left(L_{0}\right)$ can be expressed as Eq. (10) by substituting constant parameter $\lambda$ obtained by Eq. (8) into Eq. (9).

$\lambda(L)=\lambda\left(L_{0}\right)\left(\frac{L}{L_{0}}\right)^{\alpha}$

\subsubsection{Failure Rate under the Exponential Law}

When the baseline time-to-failure distribution is exponential and $\phi(L)$ follows the exponential law, we have Eqs. (11) - (14). Like Eq. (14), the failure rate at any load $L$ can be obtained as Eq. (15).

$R\left(t ; L_{0}\right)=R_{0}\left(t \phi\left(L_{0}\right)\right)=e^{-\lambda\left(t \phi\left(L_{0}\right)\right)}=e^{-\lambda t e^{L_{0} \alpha}}$

$F\left(t ; L_{0}\right)=1-R\left(t ; L_{0}\right)=1-e^{-\lambda t e^{L_{0} \alpha}}$

$f\left(t ; L_{0}\right)=\frac{d F\left(t ; L_{0}\right)}{d t}=\lambda e^{L_{0} \alpha} e^{-\lambda t e^{L_{0} \alpha}}$

$\lambda\left(L_{0}\right)=\frac{f\left(t ; L_{0}\right)}{R\left(t ; L_{0}\right)}=\lambda e^{L_{0} \alpha}$

$\lambda(L)=\frac{f(t ; L)}{R(t ; L)}=\lambda e^{L \alpha}$

Further, Eq. (16) can be obtained to express $\lambda(L)$ in terms of $\lambda\left(L_{0}\right)$ by substituting the constant parameter $\lambda$ obtained by Eq. (14) into Eq. (15).

$\lambda(L)=\lambda\left(L_{0}\right) e^{\left(L-L_{0}\right) \alpha}$

Hence, when the time-to-failure follows the exponential distribution and $\phi(L)$ follows the exponential law, the failure rate under the AFTM is consistent with that under the PHM (i.e., Eq. (2)). 


\section{An Illustrative Mesh SAN}

Figure 1 gives a schematic diagram of an example mesh SAN. It contains devices including the server (denoted by $\mathrm{Sr}$ and being responsible for providing data and resources services), the storage array (denoted by SA and being responsible for data storage), and switches (denoted by Sw and enabling the information communication between the server and the storage array).

The problem is to evaluate the reliability of this example SAN, that is the probability that the server and the storage array can communicate via at least one operational path consisting of switches. The influence of loading on switches is investigated under three cases:

(i) Varying load on switch SwA1;

(ii) Identically varying load on all the four switches $\{\mathrm{SwA1}, \mathrm{SwA} 2, \mathrm{SwB} 1, \mathrm{SwB} 2\}$;

(iii) Non-identically varying load on the four switches $\{$ SwA1, SwA2, SwB1, SwB2 $\}$.

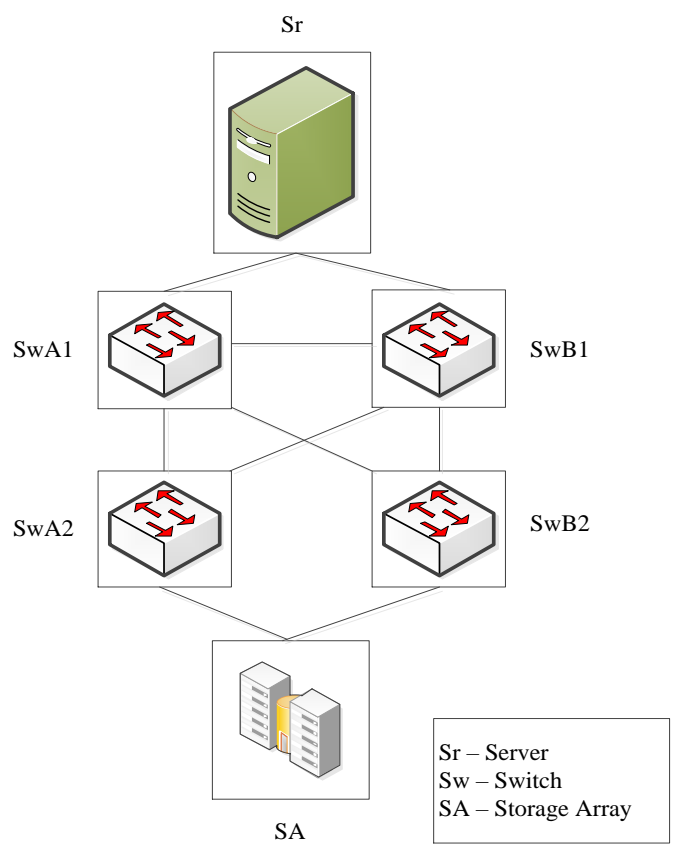

Figure 1. Example mesh SAN.

The time-to-failure of the example SAN devices is assumed to follow the exponential distribution with constant rate $\lambda$. Table 1 lists baseline failure rate parameters for the devices in the example mesh SAN, $\lambda_{i 0}(i \in\{\mathrm{Sr}, \mathrm{SA}, \mathrm{SwA} 1, \mathrm{SwA} 2, \mathrm{SwB} 1, \mathrm{SwB} 2\})$, which are based on technical specifications of products in the industry (Simache and Kaaniche, 2005; EMC Corporation, 2009; Dell EMC VMAX3, 2018).

Table 1. Baseline failure rates for components of the example mesh SAN.

\begin{tabular}{|c|c|c|c|c|c|}
\hline$\lambda_{\text {Sr0 }}$ & $\lambda_{\text {SA0 }}$ & $\lambda_{\text {SwA10 }}$ & $\lambda_{\text {SwA20 }}$ & $\lambda_{\text {SwB10 }}$ & $\lambda_{\text {SwB20 }}$ \\
\hline $1.04260401 \mathrm{e}-7$ & $4.75646981 \mathrm{e}-11$ & $4.75646981 \mathrm{e}-11$ & $4.75646981 \mathrm{e}-11$ & $4.75646981 \mathrm{e}-11$ & $4.75646981 \mathrm{e}-11$ \\
\hline
\end{tabular}




\section{SAN Reliability Modeling and Analysis}

In this section, we derive the reliability expression of the example mesh SAN based on the dynamic fault tree modeling and the subsequent binary decision diagrams-based reliability analysis.

\subsection{Fault Tree Modeling}

The failure of the example mesh SAN can be caused by one of the following causes:

- Failure of the server Sr,

- Failure of the storage array SA,

- Failure of all connections or paths between Sr and SA.

These causes are represented by the dynamic fault tree (DFT) model in Figure 2. Specifically, the server Sr fails when Sr fails itself or when both SwA1 and SwB1 fail (as the server is accessible or can communicate with other devices via one of these two switches). In other words, the server $\mathrm{Sr}$ has the functional dependence on switches SwA1 and SwB1, which is modeled by the FDEP gate. The failure of SwA1 and SwB1 serves as the trigger event of the FDEP gate, whose occurrence causes the dependent component $\mathrm{Sr}$ to become inaccessible or unusable.

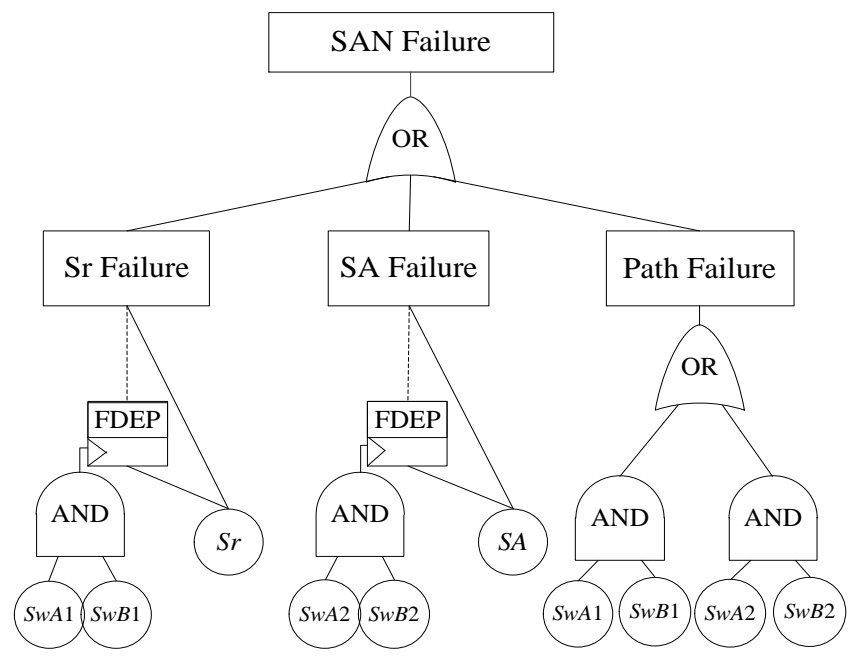

Figure 2. DFT model of the example mesh SAN.

The storage array SA fails when SA fails itself or when both SwA2 and SwB2 fail (as the storage array is accessible or can communicate with other devices via one of these two switches). In other words, the storage array SA has the functional dependence on switches SwA2 and SwB2, which is modeled by the FDEP gate, whose occurrence causes the dependent component SA to become inaccessible or unusable.

All the paths between Sr and SA fail when either the top two switches (i.e., switches SwA1 and SwB1) fail or the bottom two switches (i.e., switches SwA2 and SwB2) fail. In Figure 2, this is modeled by an OR gate connecting two AND gates.

For the system reliability analysis, the FDEP gate can be replaced with the logic OR gate (Xing et al., 2014). Thus, the DFT model in Figure 2 can be converted to the equivalent static fault tree (FT) model in Figure 3 after the OR gate replacements. 


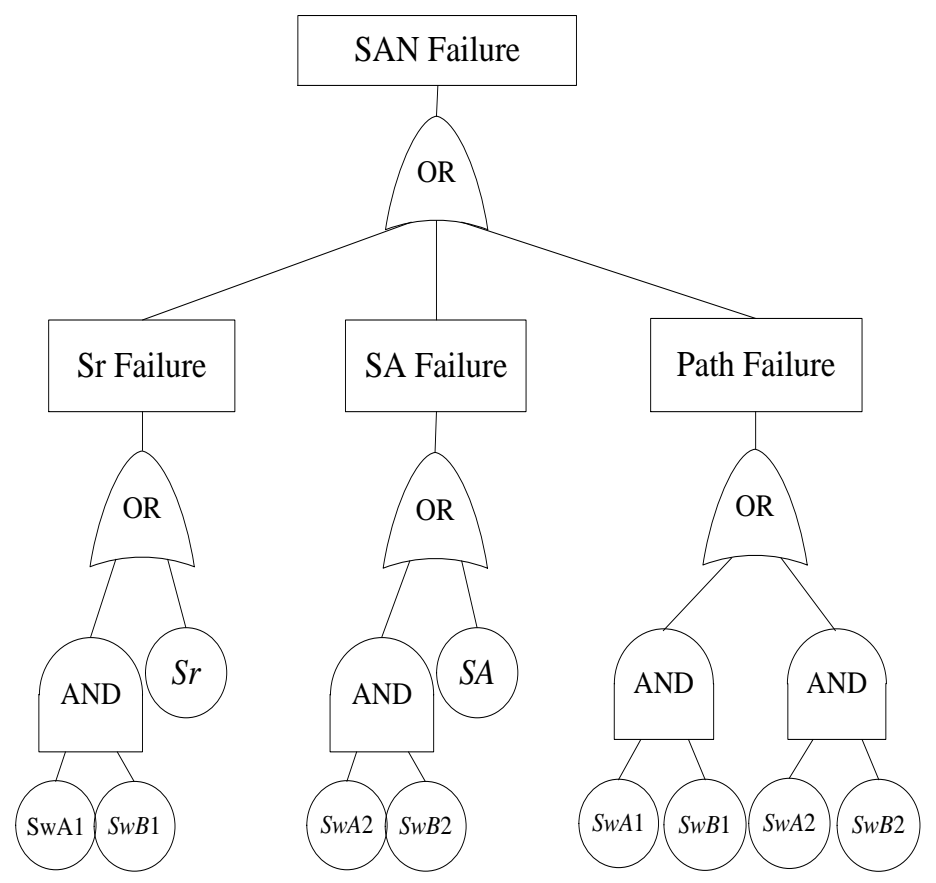

Figure 3. Equivalent FT model of the example mesh SAN.

\subsection{Binary Decision Diagram-Based Reliability Analysis}

Binary decision diagrams (BDDs) were first adapted to the fault tree reliability analysis in 1993 (Harpel et al., 1997; Xing and Dugan, 2002). A BDD is a directed acyclic graph based on Shannon's decomposition and has become a state-of-the-art combinatorial reliability model for efficient fault tree analysis since 1993. Particularly, for the reliability analysis of the example SAN, each device $i$ is modeled using a Boolean variable, denoted by $i, i \in\{\mathrm{Sr}, \mathrm{SA}, \mathrm{SwA1}, \mathrm{SwA} 2, \mathrm{SwB} 1, \mathrm{SwB} 2\} . i=0$ represents that component $i$ functions throughout the entire mission, and $i=1$ represents that component $i$ fails during the mission time. Applying the manipulation rules of the BDD generation (Eq. (3.3) in Xing and Amari (2015)), the BDD model of the entire SAN can be constructed from the FT in Figure 3 in a bottom-up manner, as shown in Figure 4. The ordering of component variables for constructing the $\mathrm{BDD}$ is $\mathrm{Sr}<\mathrm{SA}<\mathrm{SwA} 1<\mathrm{SwA} 2<\mathrm{SwB} 1<\mathrm{SwB} 2$.

Let $F_{i}$ represent component $i$ 's failure probability. The reliability of the example SAN, denoted by $R_{\text {sys }}$ is evaluated by Eq. (17), which is obtained by adding probabilities of all paths from the root node to sink node ' 0 ' of the BDD in Figure 4.

$$
\begin{aligned}
& R_{s y s}=\left(1-F_{S r}\right)\left(1-F_{S A}\right)\left\{\left(1-F_{S w A 1}\right)\left[\left(1-F_{S W A 2}\right)+F_{S w A 2}\left(1-F_{S w B 2}\right)\right]+F_{S w A 1}[(1-\right. \\
& \left.\left.\left.F_{S w A 2}\right)\left(1-F_{S w B 1}\right)+F_{S w A 2}\left(1-F_{S w B 1}\right)\left(1-F_{S w B 2}\right)\right]\right\}
\end{aligned}
$$




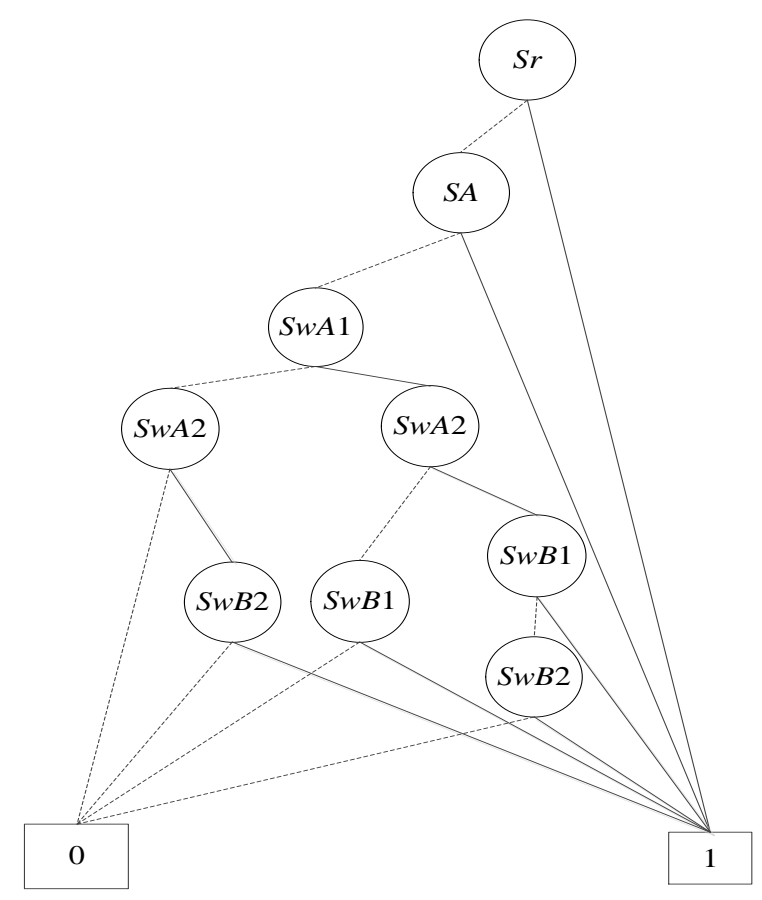

Figure 4. BDD model of the example mesh SAN.

\section{Influence of Loading on Switch Reliability and SAN Reliability}

In this section, we evaluate the switch reliability and SAN reliability using the PHM, AFTM with the power law, and AFTM with the exponential law under the three cases defined in Section 3.

\subsection{PHM}

\subsubsection{Case 1: Varying Load on SwA1}

To examine the influence of loading on SwA1 on the reliability of SwA1 and the reliability of the entire SAN system, we vary the value of $L_{\mathrm{SwA} 1}$ from 0 to 40 . We also examine the effects of the PHM load model parameter $\alpha_{\mathrm{SwA} 1}$ by analyzing the reliability of SwA1 and the entire SAN system using three different values, $\alpha_{\mathrm{SwAl}}=0.5,1.0,1.5$.

For components that have constant data loads, i.e., $\{S r, S A, S w A 2, S w B 1, S w B 2\}$, their failure probabilities are calculated as $F_{i}(t)=1-e^{-\lambda_{i 0} t}$ using the baseline failure rates $\lambda_{i 0}$ from Table 1 . Mission time $t=8640$ hours is used. Table 2 summarizes the results of $F_{i}(t=8640 h)$ for components $S r, S A, S w A 2, S w B 1, S w B 2$.

Table 2. Component unreliability $F_{i}(t=8640 \mathrm{~h}), i \in\{\mathrm{Sr}, \mathrm{SA}, \mathrm{SwA} 2$, SwB1, SwB2 $\}$.

\begin{tabular}{|c|c|c|c|c|}
\hline $\boldsymbol{F}_{\text {Sr }}$ & $\boldsymbol{F}_{\text {SA }}$ & $\boldsymbol{F}_{\text {SwA2 }}$ & $\boldsymbol{F}_{\text {SwB1 }}$ & $\boldsymbol{F}_{\text {SwB2 }}$ \\
\hline $9.00404262 \mathrm{e}-4$ & $4.10958905 \mathrm{e}-7$ & $4.10958905 \mathrm{e}-7$ & $4.10958905 \mathrm{e}-7$ & $4.10958905 \mathrm{e}-7$ \\
\hline
\end{tabular}

Table 3 summarizes the load-dependent failure rate of SwA1 ( $\lambda_{\mathrm{SwA} 1}$ evaluated using Eq. (2)), reliability of SwA1 $\left(R_{\mathrm{SwA} 1}\right.$ evaluated using $\left.e^{-\lambda_{S w A 1} t}\right)$, and reliability of the entire SAN $\left(R_{\mathrm{sys}}\right.$ evaluated using Eq. (17)) under three different load values (initial load $L_{0}=0, L=15$, and $L=40$ ) for the three different values of $\alpha_{\mathrm{SwA} 1}$. 
Table 3. Switch failure rate $\left(\lambda_{S w A I}\right)$ and reliability $\left(R_{S w A l}\right)$, and SAN reliability $\left(R_{s y s}\right)$ at $t=8640 \mathrm{~h}$.

\begin{tabular}{|c|c|c|c|c|}
\hline$\overbrace{\alpha_{S w A 1}} L$ & $\mathbf{0}$ & 15 & 40 & Metrics Evaluated \\
\hline 0.5 & $4.75646978 \mathrm{e}-11$ & $8.59989911 \mathrm{e}-8$ & $2.30767359 \mathrm{e}-2$ & \multirow{3}{*}{$\lambda_{\mathrm{SwA} 1}$} \\
\hline 1.0 & $4.75646978 \mathrm{e}-11$ & $1.55489823 \mathrm{e}-4$ & $1.11960291 \mathrm{e}+8$ & \\
\hline 1.5 & $4.75646978 \mathrm{e}-11$ & $2.81132196 \mathrm{e}-1$ & $5.43192364 \mathrm{e}+15$ & \\
\hline 0.5 & 0.99999959 & 0.99925725 & 0 & \multirow{3}{*}{$R_{\mathrm{SwA} 1}$} \\
\hline 1.0 & 0.99999959 & 0.26094854 & 0 & \\
\hline 1.5 & 0.99999959 & 0 & 0 & \\
\hline 0.5 & 0.99909919 & 0.99909912 & 0.99909878 & \multirow{3}{*}{$R_{\mathrm{sys}}$} \\
\hline 1.0 & 0.99909919 & 0.99909888 & 0.99909878 & \\
\hline 1.5 & 0.99909919 & 0.99909878 & 0.99909878 & \\
\hline
\end{tabular}

Figure 5 shows the effects of the loading on SwA1 and the load model parameter $\alpha_{\mathrm{SwA}}$ on the reliability of switch SwA1 and on the reliability of the entire example SAN graphically. At the beginning as the loading on SwA1 increases, both the reliability of SwA1 and reliability of the SAN decrease slowly. When the load of SwA1 reaches a certain value, both the individual switch reliability and the SAN reliability drop sharply, and this load value decreases as the model parameter $\alpha_{\mathrm{SwA} 1}$ increases (specifically, $L=27$ for $\alpha_{\mathrm{SwAl}}=0.5 ; L=13$ for $\alpha_{\mathrm{SwAl}}=1.0 ; L=8$ for $\alpha_{\mathrm{SwA1}}=1.5$ ). This is intuitive as the larger the value of $\alpha_{\mathrm{SwA1}}$, the more significant effects of data load on the switch failure rate (according to Eq. (2)), and thus on the switch reliability.

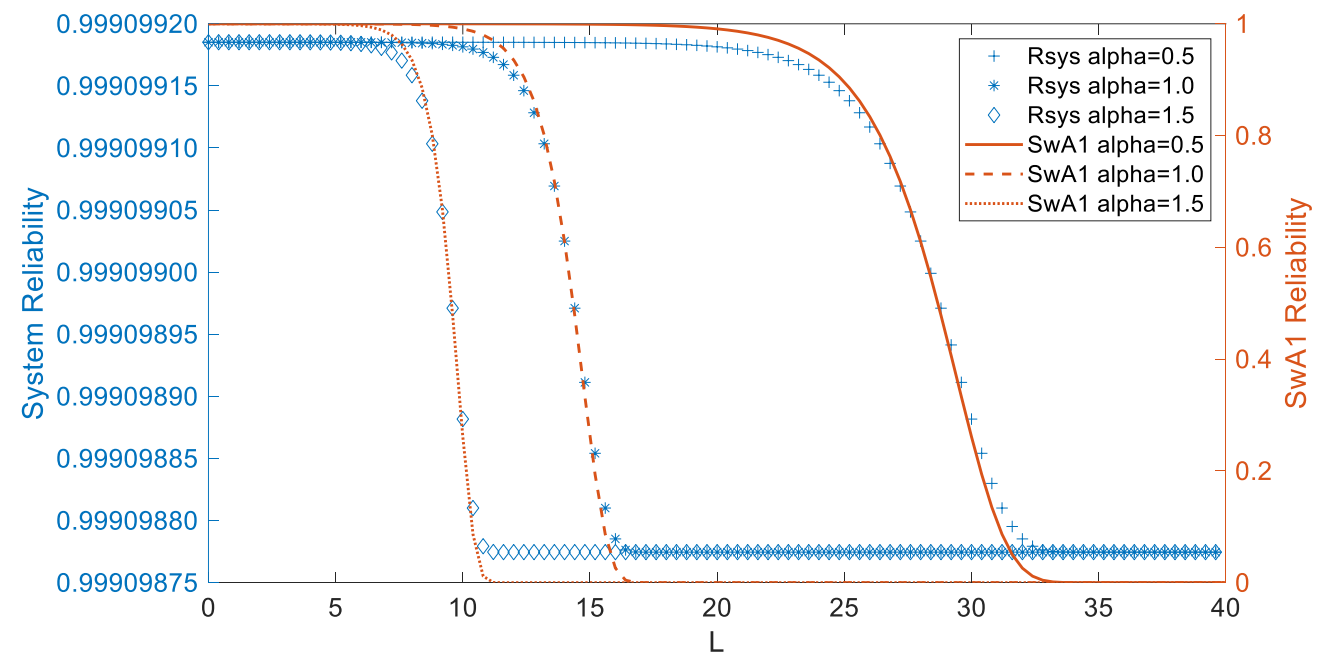

Figure 5. $R_{s y s}(L)$ and $R_{S w A l}(L)$ under different $\alpha_{S w A l}$ at $t=8640 \mathrm{~h}$.

\subsubsection{Case 2: Identically Varying Load on All the Four Switches}

To examine the influence of identically varying load of all the four switches on their reliability and the reliability of the entire SAN system, we vary the value of $L_{i}, i \in\{S w A 1, S w A 2, S w B 1, S w B 2\}$ from 0 to 40 . We also examine the effects of mission time $t$ by analyzing those reliabilities using three different values $(t=720,4320$, and 8640 hours).

For components that have constant data loads, i.e., $\{S r, S A\}$ in this case, their failure probabilities are calculated as $F_{i}(t)=1-e^{-\lambda_{i 0} t}$ using the baseline failure rates $\lambda_{i 0}$ from Table 1 . Table 4 summarizes the results of $F_{i}(t)$ for components $S r, S A$ at $t=720,4320$, and 8640 hours. 
Table 4. Component unreliability $F_{i}(t), i \in\{\mathrm{Sr}, \mathrm{SA}\}$.

\begin{tabular}{|c|c|c|}
\hline $\boldsymbol{t}(\boldsymbol{h})$ & $\boldsymbol{F}_{\mathbf{S r}}$ & $\boldsymbol{F}_{\mathbf{S A}}$ \\
\hline 720 & $7.50646716 \mathrm{e}-5$ & $3.42465818 \mathrm{e}-8$ \\
\hline 4320 & $4.50303517 \mathrm{e}-4$ & $2.05479473 \mathrm{e}-7$ \\
\hline 8640 & $9.00404262 \mathrm{e}-4$ & $4.10958905 \mathrm{e}-7$ \\
\hline
\end{tabular}

Table 5 summarizes the load-dependent failure rate of each switch $i \quad\left(\lambda_{i}, i \in\right.$ $\{S w A 1, S w A 2, S w B 1, S w B 2\}$ evaluated using Eq. (2)), reliability of each switch $i\left(R_{i}\right.$ evaluated using $e^{-\lambda_{i} t}$ ), and reliability of the entire SAN ( $R_{\text {sys }}$ evaluated using Eq. (17)) under three different load values (initial load $L_{0}=0, L=15$, and $L=20$ ) at three different mission times. The PHM load model parameter $\alpha_{i}=1, i \in\{S w A 1, S w A 2, S w B 1, S w B 2\}$ is used in this case.

Table 5. Switch failure rate $\left(\lambda_{i}\right)$ and reliability $\left(R_{i}\right)$, and SAN reliability $\left(R_{\mathrm{sys}}\right)$ for $\alpha_{i}=1$.

\begin{tabular}{|c|c|c|c|c|}
\hline $\boldsymbol{y y y n} \boldsymbol{t}(\boldsymbol{h})$ & $\mathbf{0}$ & $\mathbf{1 5}$ & $\mathbf{2 0}$ & \multirow{2}{*}{ Metrics Evaluated } \\
\hline 720 & $4.75646978 \mathrm{e}-11$ & $1.55489823 \mathrm{e}-4$ & $2.30767359 \mathrm{e}-2$ & \multirow{2}{*}{$\lambda_{i}$} \\
\hline 4320 & $4.75646978 \mathrm{e}-11$ & $1.55489823 \mathrm{e}-4$ & $2.30767359 \mathrm{e}-2$ & \multirow{2}{*}{$R_{i}$} \\
\hline 8640 & $4.75646978 \mathrm{e}-11$ & $1.55489823 \mathrm{e}-4$ & $2.30767359 \mathrm{e}-2$ & \\
\hline 720 & 0.99999997 & 0.89408657 & $6.08259182 \mathrm{e}-8$ & \multirow{2}{*}{$R_{\text {sys }}$} \\
\hline 4320 & 0.99999980 & 0.51083122 & 0 & 0 \\
\hline 8640 & 0.99999959 & 0.26094854 & $1.46549439 \mathrm{e}-14$ & 0 \\
\hline
\end{tabular}

Figure 6 shows the effects of the identically varying load of all the switches and the mission time on the switch reliability and on the entire SAN reliability graphically.

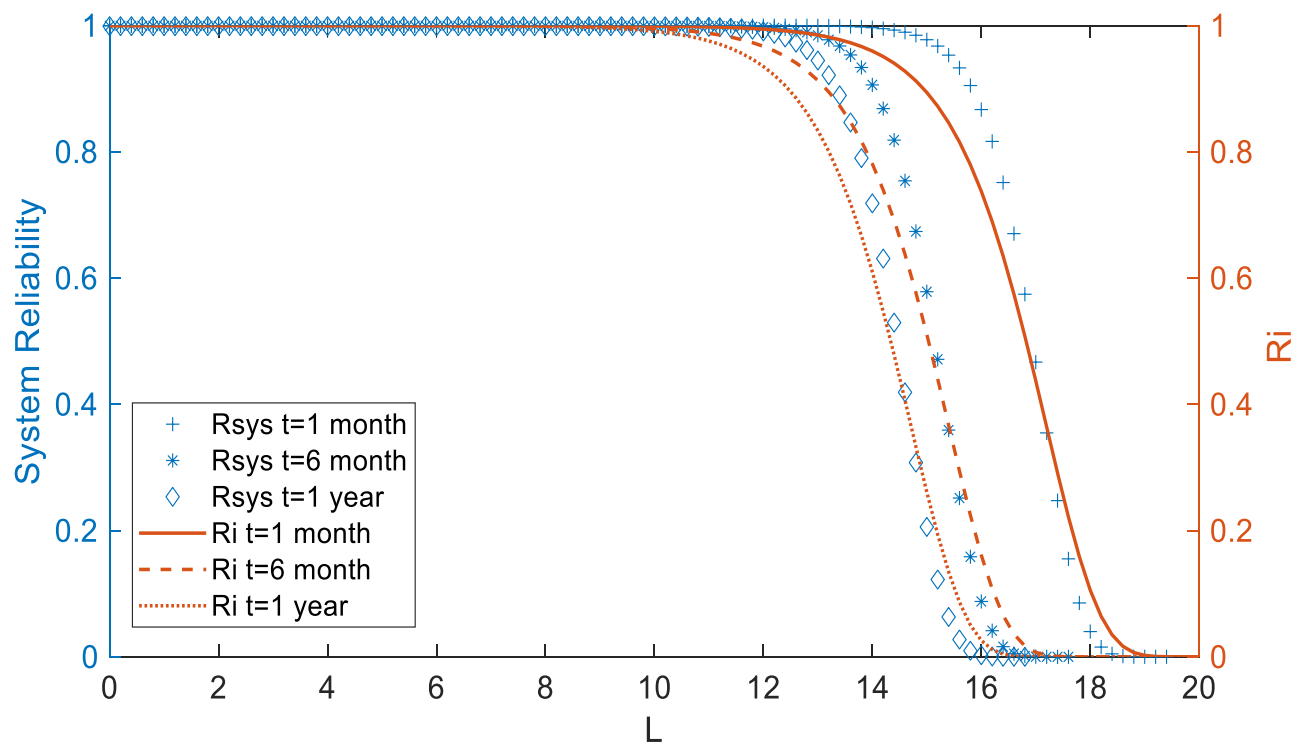

Figure 6. $R_{i}(L)$ and $R_{s y s}(L)$ at different $t$ under $\alpha_{i}=1, i \in\{S w A 1, S w A 2, S w B 1, S w B 2\}$. 
At the beginning as the loading on all switches increases, both the switch reliability and the SAN reliability decrease very slightly. When the load of the switches reaches a certain value, the switch and SAN reliabilities drop sharply, and this load value decreases as the mission time $t$ increases (specifically, $L=17$ for $t=720 \mathrm{~h}, L=15$ for $t=4320 \mathrm{~h}, L=12$ for $t=8640 \mathrm{~h}$ ). This is intuitive as the larger the value of $t$, each switch's failure probability increases, leading to the worse SAN reliability.

\subsubsection{Case 3: Non-Identically Varying Load on the Four Switches}

To examine the influence of non-identically varying load on all the four switches on their reliability and the entire SAN system reliability, we vary each $L_{i}, i \in\{S w A 1, S w A 2, S w B 1, S w B 2\}$ in different ranges of loading values. We also examine the effects of the PHM load model parameter $\alpha_{i}, i \in\{S w A 1, S w A 2, S w B 1, S w B 2\}$ by collecting the data under three different values, $\alpha_{i}=0.5,1.5$, 2.5. Table 6 summarizes the range of $L_{i}$ (the first number in the range is $L_{i 0}$ ) and value of $\alpha_{i}$ for three sets of parameters studied. Mission time $t=8640$ hours is used for this case study.

Table 6. $L_{i}$ and $\alpha_{i}, i \in\{S w A 1, S w A 2, S w B 1, S w B 2\}$.

\begin{tabular}{|c|c|c|c|c|c|}
\hline Parameter & $\boldsymbol{a}_{i}$ & $\boldsymbol{L}_{\boldsymbol{S} w \mathbf{A 1}}$ & $\boldsymbol{L}_{\boldsymbol{S} w A \mathbf{2}}$ & $\boldsymbol{L}_{\boldsymbol{S W B 1}}$ & $\boldsymbol{L}_{\boldsymbol{S} w \boldsymbol{B} \mathbf{2}}$ \\
\hline Set 1 & 0.5 & $3 \sim 43$ & $1 \sim 41$ & $5 \sim 45$ & $2 \sim 42$ \\
\hline Set 2 & 1.5 & $1 \sim 41$ & $2 \sim 42$ & $3 \sim 43$ & $5 \sim 45$ \\
\hline Set 3 & 2.5 & $5 \sim 45$ & $3 \sim 43$ & $1 \sim 41$ & $2 \sim 42$ \\
\hline
\end{tabular}

For components that have constant data loads, i.e., $\{S r, S A\}$, their failure probabilities at $t=8640 \mathrm{~h}$ are given in Table 4.

Table 7 summarizes the load-dependent failure rate of each switch $i, i \in$ $\{S w A 1, S w A 2, S w B 1, S w B 2\},\left(\lambda_{i}\right.$ evaluated using Eq. (2)) and the entire SAN reliability $\left(R_{\mathrm{sys}}\right.$ evaluated using Eq. (17)) under the initial load $L_{i 0}$ and $L_{i}=L_{i 0}+4$ and $L_{i}=L_{i 0}+40$.

Table 7. Switch failure rate $\left(\lambda_{i}\right)$ and SAN reliability $\left(R_{\text {sys }}\right)$ under different loads $L_{i}$ and $\alpha_{i}$ at $t=8640 \mathrm{~h}$.

\begin{tabular}{|c|c|c|c|c|c|c|}
\hline$L_{i}$ & $\alpha_{i}$ & $\lambda_{S_{w A 1}}$ & $\lambda_{S_{W A 2}}$ & $\lambda_{S w B 1}$ & $\lambda_{S w B 2}$ & $\boldsymbol{R}_{s y s}\left(\boldsymbol{L}_{i}\right)$ \\
\hline \multirow{3}{*}{$L_{i 0}$} & 0.5 & $2.13170186 \mathrm{e}-10$ & $7.84209290 \mathrm{e}-11$ & $5.79456643 \mathrm{e}-10$ & $1.29294254 \mathrm{e}-10$ & 0.99909919 \\
\hline & 1.5 & $2.13170186 \mathrm{e}-10$ & $9.55362494 \mathrm{e}-10$ & $4.28163765 \mathrm{e}-9$ & $8.59989911 \mathrm{e}-8$ & 0.99909918 \\
\hline & 2.5 & $1.27633819 \mathrm{e}-5$ & $8.59989910 \mathrm{e}-8$ & $5.79456644 \mathrm{e}-10$ & $7.05922706 \mathrm{e}-9$ & 0.99909862 \\
\hline \multirow{3}{*}{$L_{i 0}+4$} & 0.5 & $1.57512647 \mathrm{e}-9$ & $5.79456644 \mathrm{e}-10$ & $4.28163765 \mathrm{e}-9$ & $9.55362494 \mathrm{e}-10$ & 0.99909918 \\
\hline & 1.5 & $8.59989911 \mathrm{e}-8$ & $3.85420738 \mathrm{e}-7$ & $1.72733591 \mathrm{e}-6$ & $3.46944692 \mathrm{e}-5$ & 0.99822792 \\
\hline & 2.5 & 0.28113220 & $1.89425384 \mathrm{e}-3$ & $1.27633819 \mathrm{e}-5$ & $1.55489823 \mathrm{e}-4$ & 0.23349171 \\
\hline \multirow{3}{*}{$L_{i 0}+40$} & 0.5 & $1.03422755 \mathrm{e}-1$ & $3.80471053 \mathrm{e}-2$ & $2.81132196 \mathrm{e}-1$ & $6.27290719 \mathrm{e}-2$ & 0 \\
\hline & 1.5 & $2.43441928 \mathrm{e}+16$ & $1.09103103 \mathrm{e}+17$ & $4.88966183 \mathrm{e}+17$ & $9.82114833 \mathrm{e}+18$ & 0 \\
\hline & 2.5 & $3.43094658 \mathrm{e}+38$ & $2.31175362 \mathrm{e}+36$ & $1.55764734 \mathrm{e}+34$ & $1.89760293 e+35$ & 0 \\
\hline
\end{tabular}

Figures 7, 8 and 9 show the effects of the varying loading on the reliability of each switch $R_{i}\left(L_{i 0}+L\right)$ and on the reliability of the entire example SAN $R_{s y s}\left(L_{i 0}+L\right)$ graphically for $\alpha_{i}$ being $0.5,1.5$ and 2.5, respectively. The values of $L_{i 0}$ are given in Table 6 , and $L$ corresponds to the $x$-axis of these figures. Similar to the observation in Section 5.1.1, at the beginning, as the loading on all the switches increases, both the switch reliability and the SAN reliability decrease very slightly. When the load of each switch reaches a certain value, the switch reliability drops sharply, leading to a sudden drop in the entire SAN reliability. This load value varies for different switches. For example, in Figure 7 the reliability of switch SwB1 drops at first among the four switches, because SwB1 has the largest initial load $L_{i 0}$. 


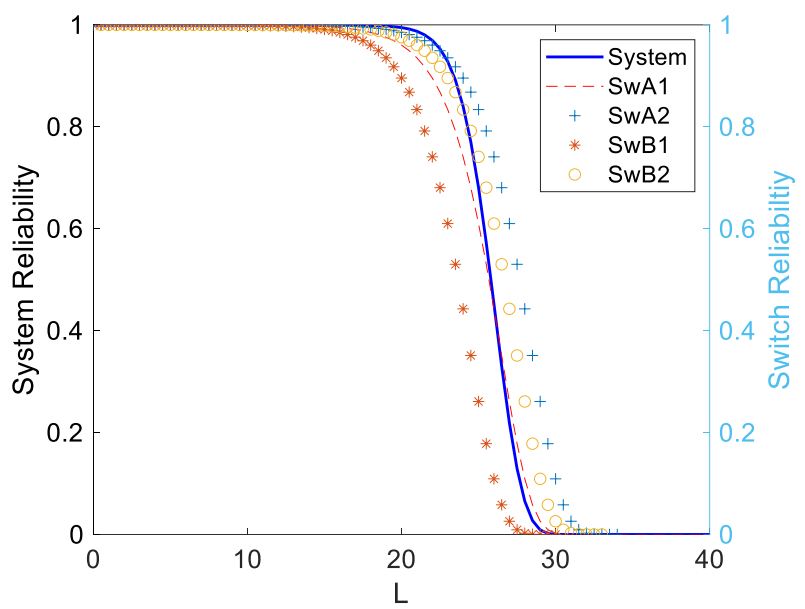

Figure 7. Set 1 in Table $6\left(L_{S w A I O}=3, L_{S w A 20}=1, L_{S w B I 0}=5, L_{S w B 20}=2\right.$, and $\left.\alpha_{i}=0.5\right)$.

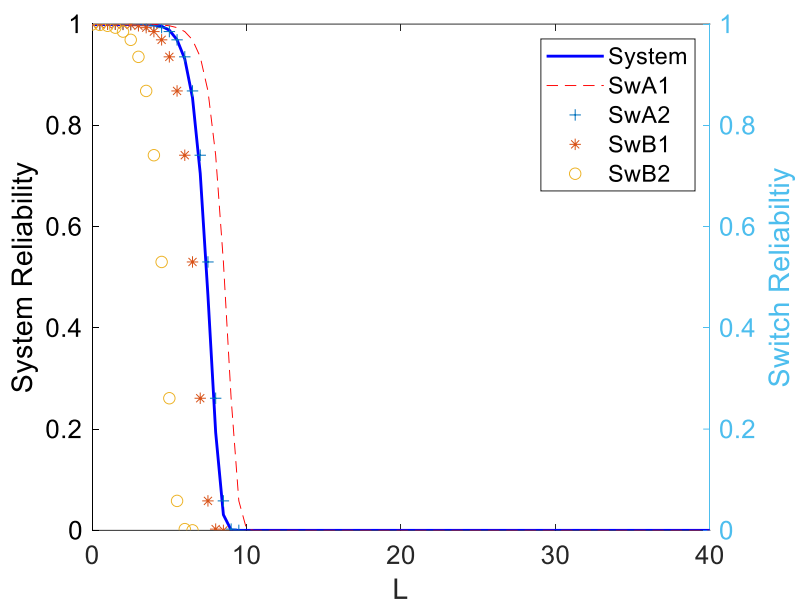

Figure 8. Set 2 in Table $6\left(L_{S w A 10}=1, L_{S w A 20}=2, L_{S w B I 0}=3, L_{S w B 20}=5\right.$, and $\left.\alpha_{i}=1.5\right)$.

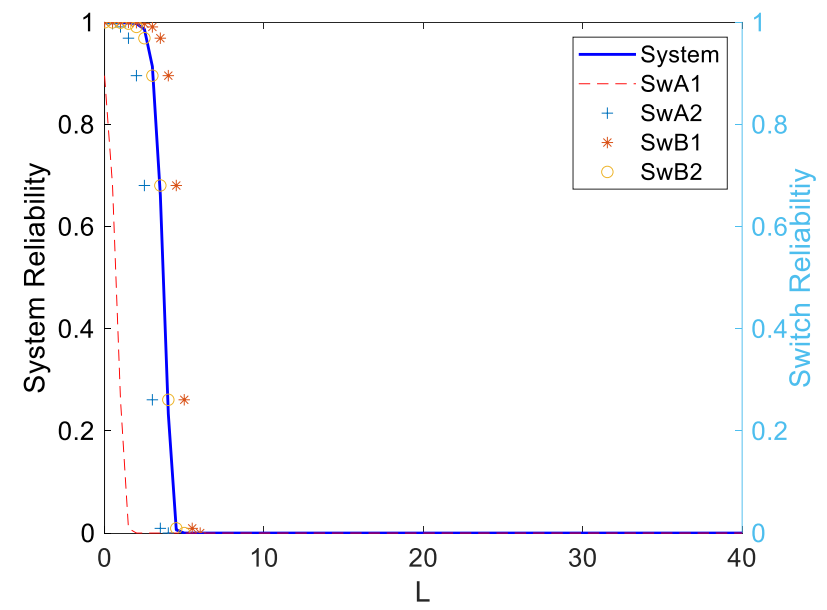

Figure 9. Set 3 in Table $6\left(L_{S w A 10}=5, L_{S w A 20}=3, L_{S w B 10}=1, L_{S w B 20}=2\right.$, and $\left.\alpha_{i}=2.5\right)$. 


\subsection{AFTM under the Power Law}

\subsubsection{Case 1: Varying Load on SwA1}

To examine the influence of loading on SwA1 on the reliability of SwA1 and the reliability of the entire SAN system, we vary the value of $L_{S w A 1}$ from 1 to 1000 . We also examine the effects of the AFTM load model parameter $\alpha_{S w A 1}$ by analyzing the reliability of SwA1 and the entire SAN system using three different values, $\alpha_{S w A 1}=2.0,2.5,3.0$. Mission time $t=8640$ hours is used.

For components that have constant data loads, i.e., $\{S r, S A, S w A 2, S w B 1, S w B 2\}$, their failure probabilities, $F_{i}(t=8640 h)$, are given in Table 2 .

Table 8 summarizes the load-dependent failure rate of SwA1 ( $\lambda_{\mathrm{SwA} 1}$ evaluated using Eq. (9)), reliability of SwA1 ( $R_{\mathrm{SwA} 1}$ evaluated using Eq. (5)), and reliability of the entire SAN $\left(R_{\text {sys }}\right.$ evaluated using Eq. (17)) at three load values (initial load $L_{0}=1, L=200$, and $L=1000$ ) for the three different values of $\alpha_{\mathrm{SwA}}$.

Table 8. Switch failure rate $\left(\lambda_{\mathrm{SwAl}}\right)$ and reliability $\left(R_{\mathrm{SwAl}}\right)$, and SAN reliability $\left(R_{\mathrm{sys}}\right)$ at $t=8640 \mathrm{~h}$.

\begin{tabular}{|c|c|c|c|c|}
\hline$a_{\alpha_{S W A 1}}^{L}$ & 1 & 200 & 1000 & Metrics Evaluated \\
\hline 2.0 & $4.75646978 \mathrm{e}-11$ & $1.90258791 \mathrm{e}-6$ & $4.75646978 \mathrm{e}-5$ & \multirow{3}{*}{$\lambda_{\mathrm{SwA} 1}$} \\
\hline 2.5 & $4.75646978 \mathrm{e}-11$ & $2.69066563 e-5$ & $1.50412781 \mathrm{e}-3$ & \\
\hline 3.0 & $4.75646978 \mathrm{e}-11$ & $3.80517582 \mathrm{e}-4$ & $4.75646978 \mathrm{e}-2$ & \\
\hline 2.0 & 0.99999959 & 0.98369601 & 0.66301412 & \multirow{3}{*}{$R_{\mathrm{SwA} 1}$} \\
\hline 2.5 & 0.99999959 & 0.79257074 & $2.27015078 \mathrm{e}-6$ & \\
\hline 3.0 & 0.99999959 & 0.03734068 & 0 & \\
\hline 2.0 & 0.99909919 & 0.99909918 & 0.99909905 & \multirow{3}{*}{$R_{\mathrm{sys}}$} \\
\hline 2.5 & 0.99909919 & 0.99909910 & 0.99909878 & \\
\hline 3.0 & 0.99909919 & 0.99909879 & 0.99909878 & \\
\hline
\end{tabular}

Figure 10 shows the effects of the loading on SwA1 and the load model parameter $\alpha_{\mathrm{SwA}}$ on the reliability of switch SwA1 and on the reliability of the entire example SAN graphically. It is intuitive that the switch reliability and the SAN reliability decreases as the loading on SwA1 increases. The decreasing trend is the sharpest when $\alpha_{\mathrm{SwA} 1}=3$ among the three values studied for this parameter. This is also intuitive since as the value of $\alpha_{\mathrm{SwA} 1}$ increases, the effects of data load on the switch failure rate (according to Eq. (9)) and on the switch reliability (according to Eq. (5)) and thus on the entire SAN reliability become more prominent.

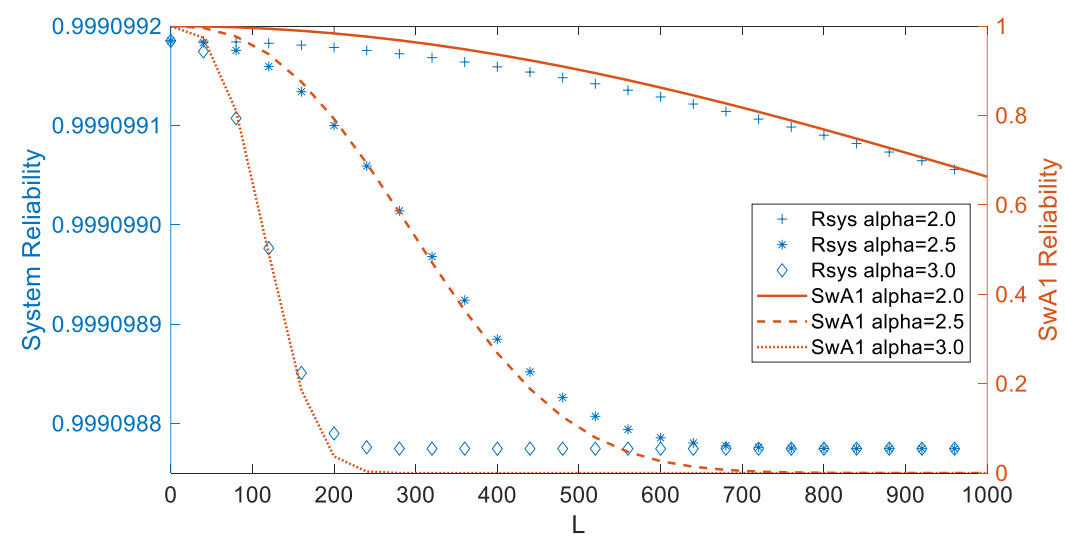

Figure 10. $R_{\text {sys }}(L)$ and $R_{S w A l}(L)$ under different $\alpha_{S w A l}$ at $t=8640 \mathrm{~h}$. 


\subsubsection{Case 2: Identically Varying Load on All the Four Switches}

To examine the influence of identically varying load on all four switches on the switch reliability and the SAN reliability, we vary the value of $L_{i}(i \in\{S w A 1, S w A 2, S w B 1, S w B 2\})$ from 1 to 1000. We also examine the effects of mission time $t$ by analyzing those reliabilities using three different values $(t=720,4320$, and 8640 hours).

For components that have constant data loads, i.e., $\{S r, S A\}$ in this case, their failure probabilities at $t=720,4320$, and 8640 hours are summarized in Table 4 .

Table 9 summarizes the load-dependent failure rate of each switch $i \quad\left(\lambda_{i}, i \in\right.$ $\{S w A 1, S w A 2, S w B 1, S w B 2\}$ evaluated using Eq. (9)), reliability of each switch $i\left(R_{i}\right.$ evaluated using Eq. (5)), and reliability of the entire SAN ( $R_{\text {sys }}$ evaluated using Eq. (17)) at three different load values (initial load $L_{0}=0, L=200$, and $L=1000$ ) for the three different values of $t$. The power law model parameter $\alpha_{i}=3, i \in\{S w A 1, S w A 2, S w B 1, S w B 2\}$ is used in this case.

Figure 11 shows the effects of the identically varying load on all four switches and the mission time on the switch reliability and on the entire SAN reliability graphically. When the load of switch $i$ increases, both the switch reliability and the SAN reliability drop, and the dropping trend is sharper as mission time $t$ increases. This is because, as $t$ increases, each switch's reliability decreases (according to Eq. (5)), thus reducing the entire SAN's reliability.

Table 9. Switch failure $\left(\lambda_{i}\right)$ rate and reliability $\left(R_{i}\right)$, and SAN reliability $\left(R_{\text {sys }}\right)$ at $\alpha_{i}=3$.

\begin{tabular}{|c|c|c|c|c|}
\hline \multicolumn{1}{|c|}{$\boldsymbol{L}(\boldsymbol{h})$} & $\mathbf{1}$ & $\mathbf{2 0 0}$ & $\mathbf{1 0 0 0}$ & \multirow{2}{*}{ Metrics Evaluated } \\
\hline 720 & $4.75646978 \mathrm{e}-11$ & $3.80517582 \mathrm{e}-4$ & $4.75646978 \mathrm{e}-2$ & \multirow{2}{*}{$\lambda_{i}$} \\
\hline 4320 & $4.75646978 \mathrm{e}-11$ & $3.80517582 \mathrm{e}-4$ & $4.75646978 \mathrm{e}-2$ & \multirow{2}{*}{$R_{i}$} \\
\hline 8640 & $4.75646978 \mathrm{e}-11$ & $3.80517582 \mathrm{e}-4$ & $4.75646978 \mathrm{e}-2$ & \\
\hline 720 & 0.99999997 & 0.76035286 & $1.33226763 \mathrm{e}-15$ & \multirow{2}{*}{$R_{\text {sys }}$} \\
\hline 4320 & 0.99999980 & 0.19323737 & 0 & \\
\hline 8640 & 0.99999959 & 0.03734068 & 0 & \\
\hline 420 & 0.99992490 & 0.88837007 & 0 & \\
\hline
\end{tabular}

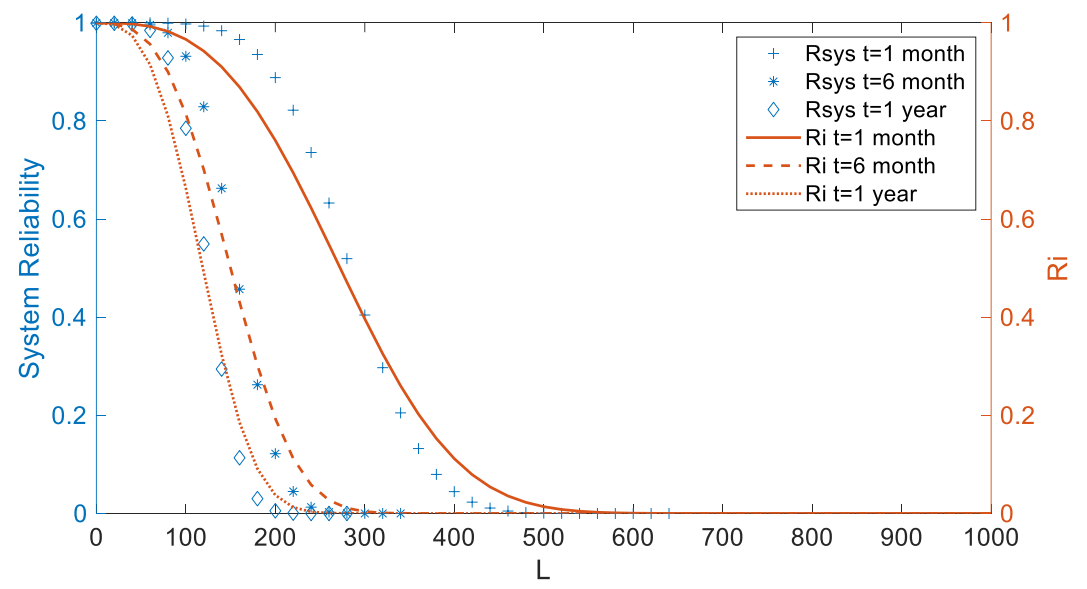

Figure 11. $R_{i}(L)$ and $R_{s y s}(L)$ at different $t$ for $\alpha_{i}=3, i \in\{S w A 1, S w A 2, S w B 1, S w B 2\}$. 


\subsubsection{Case 3: Non-Identically Varying Load on the Four Switches}

To examine the influence of non-identically varying load on all the four switches on their reliability and the entire SAN system reliability, we vary each $L_{i}, i \in\{S w A 1, S w A 2, S w B 1, S w B 2\}$, in different ranges of loading values. We also examine the effects of the power law load model parameter $\alpha_{i}, i \in\{S w A 1, S w A 2, S w B 1, S w B 2\}$, by collecting the data under three different values, $\alpha_{i}=2.0,2.5$, and 3.0. Table 10 presents the ranges of $L_{i}$ (the first number in each range is $L_{i 0}$ ) and values of $\alpha_{i}$ for three sets of parameters studied in this case. Mission time $t=8640$ hours is used for this case study.

Table 10. $L_{i}$ and $\alpha_{i}, i \in\{S w A 1, S w A 2, S w B 1, S w B 2\}$.

\begin{tabular}{|c|c|c|c|c|c|}
\hline Parameter & $\alpha_{i}$ & $L_{S w A 1}$ & $L_{S w A 2}$ & $L_{S w B 1}$ & $L_{S W B 2}$ \\
\hline Set 1 & 2.0 & $30 \sim 1030$ & $50 \sim 1050$ & $100 \sim 1100$ & $180 \sim 1180$ \\
\hline Set 2 & 2.5 & $50 \sim 1050$ & $100 \sim 1100$ & $180 \sim 1180$ & $30 \sim 1030$ \\
\hline Set 3 & 3.0 & $180 \sim 1180$ & $50 \sim 1050$ & $30 \sim 1030$ & $100 \sim 1100$ \\
\hline
\end{tabular}

For components that have constant data loads, i.e., $\{S r, S A\}$, their failure probabilities at $t=8640$ hours are given in Table 2 .

Table 11 summarizes each switch's reliability $\left(R_{i}, i \in\{S w A 1, S w A 2, S w B 1, S w B 2\}\right.$, evaluated using Eq. (5)) and the entire SAN reliability $\left(R_{\text {sys }}\right.$ evaluated using Eq. (17)) under the initial load $L_{i 0}, L_{i}=L_{i 0}+200$, and $L_{i}=L_{i 0}+1000$.

Table 11. Switch reliability $\left(R_{i}\right)$ and SAN reliability $\left(R_{\text {sys }}\right)$ under different loads $L_{i}$ and $\alpha_{i}$ at $t=8640 \mathrm{~h}$.

\begin{tabular}{|c|c|c|c|c|c|c|}
\hline$L_{i}$ & $\alpha$ & $R_{S w A 1}$ & $R_{S_{w A 2}}$ & $\boldsymbol{R}_{S w B_{1}}$ & $\boldsymbol{R}_{S w B_{2}}$ & $\boldsymbol{R}_{s y s}$ \\
\hline \multirow{3}{*}{$L_{i 0}$} & 2.0 & 0.99963021 & 0.99897313 & 0.99589884 & 0.98677318 & 0.99908410 \\
\hline & 2.5 & 0.99276153 & 0.95973709 & 0.83640659 & 0.99797623 & 0.99783477 \\
\hline & 3.0 & 0.09101665 & 0.94992725 & 0.98896544 & 0.66301412 & 0.97238848 \\
\hline \multirow{3}{*}{$L_{i 0}+200$} & 2.0 & 0.97849487 & 0.97464212 & 0.96368933 & 0.94238397 & 0.99686046 \\
\hline & 2.5 & 0.66623387 & 0.52696473 & 0.31449162 & 0.71913992 & 0.66813893 \\
\hline & 3.0 & $1.60915503 \mathrm{e}-10$ & $1.62664738 \mathrm{e}-3$ & $6.73701709 \mathrm{e}-3$ & $1.51745222-5$ & $1.10508524 \mathrm{e}-5$ \\
\hline \multirow{3}{*}{$L_{i 0}+1000$} & 2.0 & 0.65209715 & 0.64114910 & 0.61369306 & 0.56974764 & 0.73129737 \\
\hline & 2.5 & $5.95135720 \mathrm{e}-7$ & $9.98108882 \mathrm{e}-8$ & $4.39599113 \mathrm{e}-9$ & $1.17384441 \mathrm{e}-6$ & $7.62945263 \mathrm{e}-13$ \\
\hline & 3.0 & 0 & 0 & 0 & 0 & 0 \\
\hline
\end{tabular}

Figures 12, 13 and 14 show the effects of the varying loading on the reliability of each switch $R_{i}\left(L_{i 0}+L\right)$ and on the reliability of the entire example SAN $R_{s y s}\left(L_{i 0}+L\right)$ graphically for $\alpha_{i}$ being 2.0, 2.5, and 3.0, respectively. The values of $L_{i 0}$ are given in Table 10, and $L$ corresponds to the $\mathrm{x}$-axis of these figures. Similar to the observation in Section 5.2.1, as the loading on the switch increases, both the switch reliability and the SAN reliability decrease. Particularly, the reliability of switch SwB1 with the largest initial load $L_{i 0}$ drops at first among the four switches, as demonstrated in Figure 13. 


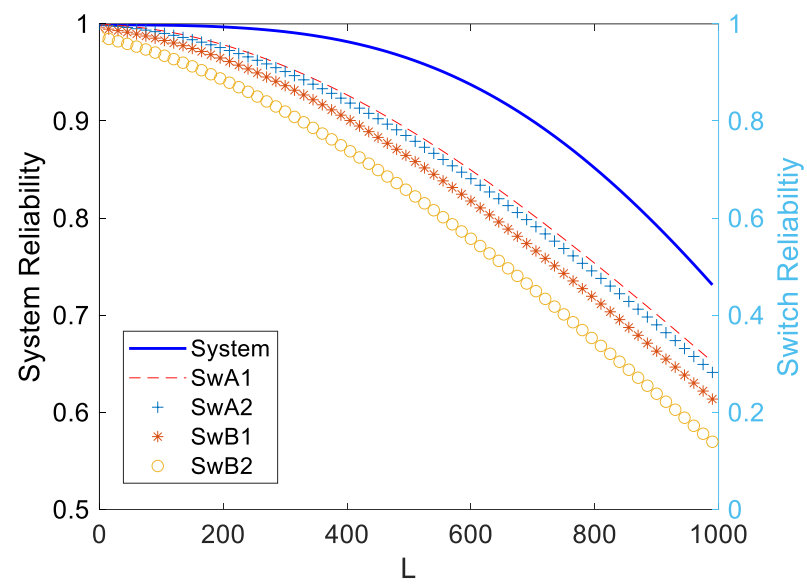

Figure 12. Set 1 in Table $10\left(L_{S w A I 0}=30, L_{S w A 20}=50, L_{S w B 10}=100\right.$, and $L_{S w B 20}=180$ and $\left.\alpha=2.0\right)$.

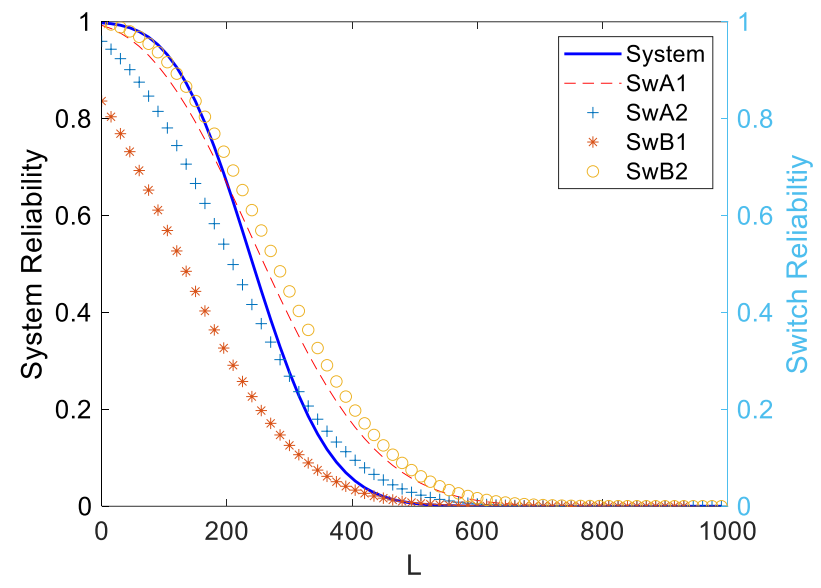

Figure 13. Set 2 in Table $10\left(L_{S w A I 0}=50, L_{S w A 20}=100, L_{S w B 10}=180\right.$, and $L_{S w B 20}=30$ and $\left.\alpha=2.5\right)$.

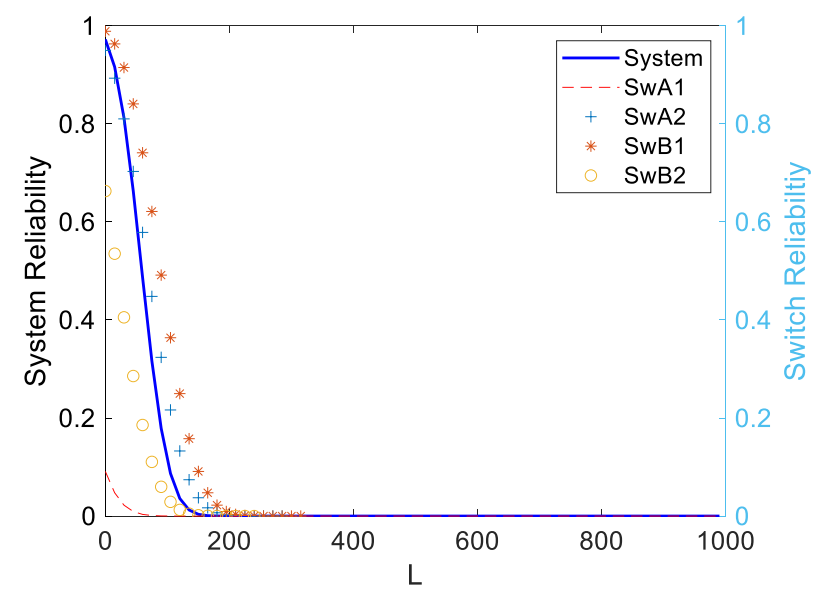

Figure 14. Set 3 in Table $10\left(L_{S w A I 0}=180, L_{S w A 20}=50, L_{S w B 10}=30\right.$, and $L_{S w B 20}=100$ and $\left.\alpha=3.0\right)$. 


\subsection{AFTM under the Exponential Law}

As discussed in Section 2.2.2, the failure rate under the AFTM with the exponential law is consistent with that under the PHM. Hence, when the same input load parameters are used, the same switch and system reliabilities as those in Section $5.1 \mathrm{can}$ be obtained. Thus, the same effects of switch loading on the SAN reliability as those in Section 5.1 can be observed, which are summarized as follows.

(i) As the loading on a single switch (e.g., SwA1) increases, both the reliability of that switch and reliability of the entire SAN decrease slowly at the beginning, then drop sharply (Figure 5). The load value corresponding to the turning point in the reliabilities decreases as the model parameter $\alpha$ increases.

(ii) As the loading on all the four switches increases, the switch reliability and the SAN reliability have similar trends to those under the varying loading on a single switch (decreasing slowly first and then dropping sharply). The load value corresponding to the turning point in the reliabilities decreases as the mission time $t$ increases (Figure 6).

(iii) In the case of switches with different initial loads, as the loading of each switch increases, the reliability of the switch with the largest initial load drops rapidly first (Figures 7, 8 and 9).

\section{Conclusions and Future Work}

Overload is one of the most common causes of cascading failures. To defend against cascading failures or at least mitigate their consequences, it is pivotal to study the impact of loading on the reliability of critical technological devices and systems. This paper contributes by modeling the effects of data loading on the switch reliability in SANs though the PHM, the AFTM with the power law, and the AFTM with the exponential law. Using the FT and BDD-based method, the effects of switch loading on the SAN reliability are further investigated. The quantitative influence of switch loading and load model parameters on the switch reliability and the SAN reliability is analyzed under three cases: varying loading on a single switch, identically and non-identically varying load on multiple switches. The results obtained from those case studies can provide effective guidelines in designing resilience strategies against switch overloading and the further cascading failures caused by the overloading. For example, before the load of a switch reaches the point where the switch reliability and the entire SAN reliability start to drop quickly (i.e., high risk of the system failure), the load of that switch may be redistributed to other devices to mitigate such risk. Thus, as a continuation of the proposed work, we are interested in exploring and developing different load redistribution policies and studying their performance in reducing the likelihood of cascading failures (Wang et al., 2008). We are also interested in studying resilience metrics, modeling and analysis of SANs subject to cascading failures (Xing, 2021).

\section{Conflict of Interest}

The authors confirm that there is no conflict of interest to declare for this publication.

\section{Acknowledgments}

This research did not receive any specific grant from funding agencies in the public, commercial, or not-for-profit sectors. The authors would like to thank the editor and anonymous reviewers for their comments that help improve the quality of this work. 


\section{References}

Amazon. (2020). Summary of the Amazon s3 service disruption in the northern Virginia (US-east-1) region. In Amazon Web Services. Available: https://aws.amazon.com/message/41926. Accessed in September 2021.

Bright, L., \& Raschid, L. (2000, August). Efficient remote data access in a mobile computing environment. In Proceedings of International Workshop on Parallel Processing (pp. 57-64). IEEE. Toronto, ON, Canada. DOI: 10.1109/ICPPW.2000.869088.

Chen, X., Qiu, J., Reedman, L., \& Dong, Z.Y. (2019). A statistical risk assessment framework for distribution network resilience. IEEE Transactions on Power Systems, 34(6), 4773-4783.

Dale, C.J. (1985). Application of the proportional hazards model in the reliability field. Reliability Engineering, 10(1), 1-14.

DELL EMC VMAX3. (2018, May). Family: Enterprise Data Services Platform for Mission Critical Hybrid Cloud and Hyper-Consolidation, Data Sheet, DELL EMC Corporation, USA. [Online]. https://www.delltechnologies.com/asset/en-us/products/storage/industry-market/h13904-vmax3embedded-nas-technical-note.pdf. Accessed in September 2021.

EMC Corporation. (2009). EMC connectrix ED-DCX-4S-B. Available: https://www.delltechnologies.com/asset/en-us/products/storage/technical-support/docu8498.pdf. Accessed in September 2021. Hopkinton, MA, USA.

Gibson, G.A., \& Rodney V.M. (2000). Network attached storage architecture. Communications of the ACM, 43(11), 37-45.

Harpel, B.M., Dugan, J.B., Walker, I.D., \& Cavallaro, J.R. (1997, January). Analysis of robots for hazardous environments. In Proceedings of Annual Reliability and Maintainability Symposium (pp.111-116). IEEE. Philadelphia, PA, USA.

Honma, S., Morishima, H., Tsukiyama, T., Matsushima, H., Oeda, T., \& Tomono, Y. (2004, April). Computer system using a storage area network and method of handling data in the computer system. Available: https://www.google.com/patents/US20040073677. Accessed in September 2021.

Hutanu, A., Allen, G., \& Kosar, T. (2010, October). High-performance remote data access for remote visualization. In Proceedings of the 11th IEEE/ACM International Conference on Grid Computing (pp. 121-128). IEEE. Brussels, Belgium.

Jiang, M., Zhou, J., \& Hu, M. (2007, August). Fuzzy reliability analysis of an iSCSI-based fault tolerant storage system organization. In Proceedings of Fourth International Conference on Fuzzy Systems and Knowledge Discovery (FSKD 2007) (Vol. 4, pp. 598-602). IEEE. Haikou, China.

Katal, A., Gupta, N., Sharma, S., \& Goudar, R.H. (2012, March). Information storage on the cloud: a survey of effective storage management system. In 2012 Students Conference on Engineering and Systems (pp. 1-6). IEEE. Allahabad, India

Kay, R., \& Kinnersley, N. (2002) On the use of the accelerated failure time model as an alternative to the proportional hazards model in the treatment of time to event data: a case study in influenza. Drug Information Journal, 36(3), 571-579.

Khanal, S.P., Sreenivas, V., \& Acharya, K.S. (2014) Accelerated failure time models: an application in the survival of acute liver failure patients in India. International Journal of Science and Research, 3(6), 161166.

Lane, W.R., Looney, S.W., \& Wansley, J.W. (1986) An application of the cox proportional hazards model to bank failure. Journal of Banking \& Finance, 10(4), 511-531. 
Levens, S. (2021, January). What's the Diff: NAS vs. SAN https://www.backblaze.com/blog/whats-the-diffnas-vs-san. Accessed in September 2021.

Levitin, G., \& Amari, S.V. (2009). Optimal load distribution in series-parallel systems. Reliability Engineering and System Safety, 94(2), 254-60.

Lin, D.Y., Wei, L.J., Ying, Z. (1998). Accelerated failure time models for counting processes. Biometrika, 85(3), 605-618.

Mishra, S., Anderson, K., Miller, B., Boyer, K., \& Warren, A. (2020). Microgrid resilience: a holistic approach for assessing threats, identifying vulnerabilities, and designing corresponding mitigation strategies. Applied Energy, 264, 114726.

Mohammad, R., Kalam, A., \& Amari, S.V. (2013, January) Reliability of load-sharing systems subject to proportional hazards model. In 2013 Proceedings of Annual Reliability and Maintainability Symposium (RAMS) (pp. 1-5). IEEE. Orlando, FL, USA.

Qiu, X., Telikepalli, R., Drwiega, T., \& Yan, J. (2005). Reliability and availability assessment of storage area network extension solutions. IEEE Communications Magazine, 43(3), 80-85.

Shetty, S. (2002, August). Determining the availability and reliability of storage configurations. In Power Solutions. http://www2.latech.edu/ box/hapc/docs/dell_storage_availablity_reliability.pdf. Accessed in September 2021.

Simache, C., \& Kaaniche, M. (2005, December). Availability assessment of sunOS/solaris unix systems based on syslogd and wtmpx log files: A case study. In 11th Pacific Rim International Symposium on Dependable Computing (PRDC'05) (pp. 8-pp). IEEE. Hunan, China.

Singh, R., \& Mukhopadhyay, K. (2011). Survival analysis in clinical trials: basics and must know areas. Perspectives in Clinical Research, 2(5), 145-148.

Timashev, S.A. (2019, February). Cyber reliability, resilience, and safety of physical infrastructures. In IOP Conference Series: Materials Science and Engineering (Vol. 481, No. 1, p. 012009). IOP Publishing. DOI:10.1088/1757-899X/481/1/012009.

Uwaechia, A.N., \& Akinsanmi, O. (2013). Reliability assessment on the performance model of Ahmadu Bello University data network repositories for storage area network design. International Journal of Innovative Research in Science, Engineering and Technology, 2(7), 3311-3315.

Wang, J., Rong, L., Zhang, L., \& Zhang, Z. (2008). Attack vulnerability of scale-free networks due to cascading failures. Physica A: Statistical Mechanics and its Applications, 387(26), 6671-6678.

Xiao, H., \& Yeh, E.M. (2011, June). Cascading link failure in the power grid: a percolation-based analysis. In Proceedings of IEEE International Conference on Communications Workshops (ICC) (pp. 1-6). IEEE. Kyoto, Japan.

Xing, L. (2021). Cascading failures in internet of things: review and perspectives on reliability and resilience. IEEE Internet of Things Journal, 8(1), 44-64.

Xing, L., \& Amari, S.V. (2015). Binary decision diagrams and extensions for system reliability analysis. John Wiley \& Sons, New Jersey.

Xing, L., \& Dugan, J.B. (2002). Analysis of generalized phased mission system reliability, performance and sensitivity. IEEE Transactions on Reliability, 51(2), 199-211.

Xing, L., Morrissette, B.A., \& Dugan, J.B. (2014). Combinatorial reliability analysis of imperfect coverage systems subject to functional dependence. IEEE Transaction on Reliability, 63(1), 367-382.

Xing, L., Tannous, M., Vokkarane, V.M., Wang, H., \& Guo J. (2017). Reliability modeling of mesh storage area networks for Internet of things. IEEE Internet of Things Journal, 4(6), 2047-2057. 
Yang, S., Zhang, J., \& Lu, D. (2016). Prediction of cascading failures in spatial networks. PLoS One, 11(4), e0153904. 